\title{
Comparative Evaluation of the Anti-ulcer Activity of Curcumin and Omeprazole during the Acute Phase of Gastric Ulcer
}

\author{
—Efficacy of Curcumin in Gastric Ulcer Prevention against Omeprazole
}

\section{Karolin Kamel Abdul-Aziz}

Zoology Department, Faculty of Science, Damanhour University, Damanhour, Egypt.

Email:k.kamel2002@yahoo.com

Received February $28^{\text {th }}, 2011$; revised July $5^{\text {th }}, 2011$; accepted July $12^{\text {th }}, 2011$.

\begin{abstract}
We have confirmed in our laboratory the antiulcer activity of curcumin during the acute chronic phase of gastric ulcer disease at doses of 20, 40 and $80 \mathrm{mg} / \mathrm{kg}$ (dissolved in saline solution). In the previous study, the potent effective dose of curcumin was $80 \mathrm{mg} / \mathrm{kg}$ that appears a propitious protective effect against gastric ulcer development. Therefore, the comparison between such recommended dose of curcumin and one of the proton pump inhibitors (PPIs) staff is worthwhile. Since, the pharmacological control of gastric acid secretion is the main desired goal for gastro-cytoprotection, particularly, the $\mathrm{H}^{+} / \mathrm{K}^{+}$-ATPase (acid proton pump) inhibitors. Nevertheless, several studies have indicated that long-term inhibition of gastric acid secretion results in mucosal hyperplasia and carcinoid tumor development, due to increase circulating gastrin levels. Ulcer and the preventive indexes were scored, mucin, juice volume, total acidity, luminal haemoglobin, total antioxidant and total peroxide were evaluated. The pro-inflammatory cytokine IL-6 and the major angiogenic growth factor VEGF levels were measured. Conclusion, curcumin and omeprazole are potentially preventing gastric lesions development in the gastric wall during the acute phase of gastric ulcer diseases, but curcumin was more potent in its effect. Curcumin promotes gastric ulcer prevention/healing by induction of angiogenesis in the granular tissue of ulcers. That may be via upregulation of VEGF expression as reflected from VEGF level in serum and gastric juice, however, omeprazole might be has no role in this story.
\end{abstract}

Keywords: Curcumin, Proton Pump Inhibitors, Total Antioxidant, Copper Nicotinate, Total Peroxide, DNA Fragmentation

\section{Introduction}

The prevention of gastric ulcer pathogenesis or its recurrence is the main desired goal for the clinical and the experimental studies in the present era. Gastric acid hypersecretion is one of the major pathogenic factors for the induction of gastric ulcer disease. Furthermore, luminal acid interferes with the process of restitution, resulting in the conversion of superficial injury to deeper mucosal lesion and inactivates the acid-labile growth factors important for maintenance of mucosal integrity and repair of superficial injury [1]. Impairment of gastric ulcer healing depends upon the increased release of proinflammatory cytokines and a decrease in the gastric mucosal blood flow and angiogenesis through reducing vascular endothelial cell growth factor (VEGF) expression [2]. Mucins are heavily glycosylated glycoproteins that are the major components of the mucus viscous gel covering epithelial tissues. They form lubricants protective selective barrier on epithelial surfaces, and modulate cell-cell and cell-extracellular matrix interactions, lymphocyte trafficking and anti-immune recognition. Their expression is regulated by several cytokines and local and endocrine hormones [3-5]. It was postulated that the increased occurrence of apoptosis in the gastric epithelium might be of pathophysiological importance in the development of stress lesions. The gastric blood flow was significantly reduced (approximately $70 \%$ of intact mucosa) immediately after water immersion and restraint stress [6]. Previous studies have suggested that the bal- 
ance between cell apoptosis and cell proliferation is of great importance for maintaining gastric mucosa integrity [7]. Under normal physiological conditions, the balance between gastric epithelial cell proliferation and death is of great importance in maintaining gastric mucosal integrity. Disturbance of this balance could result in either cell loss, leading to mucosal damage and ulcer formation, or cell accumulation, leading to cancer development [8]. Proton pup inhibitors (PPIs) are widely used for a long term in the treatment of gastric ulcer diseases. PPIs are rapidly absorbed; highly protein bound, peak absorption occurs 3 to 4 hours after oral administration, and the plasma levels are undetectable by about 11 hours after a single dose of the drug where it is extensively metabolized in the liver by the cytochrome P-450 system (particularly CYP2C19 and CYP3A4). Finally, their sulfated metabolites are excreted in the urine or feces. Their plasma half-lives are about 1 to 2 hours, but their durations of action are much longer. The bioavailability of omeprazole increases with repeated doses up to about four days, probably as a result of increasing drug absorption as intragastric acidity decreases. Omeprazole is an effective agent in the treatment of peptic ulcer disease and reflux esophagitis. Omeprazole inhibits the hepatic microsomal P-450 monooxygenase system, and the plasma half-life of drugs metabolized by this route may be extended [9]. The antisecretory effect of omeprazole results in an elevation of serum gastrin concentrations, which in humans appear to be related to the degree of acid suppression. However, toxicologic studies in the rat in which massive doses of omeprazole have been used have shown markedly elevated gastrin levels associated with ECL-cell hyperplasia and gastric carcinoid tumors, which have been found after long-term treatment.

Curcuma longa, has been used in traditional remedy for a wide range of ailments, including wound healing, urinary tract infection, and liver ailments [10]. Although, curcumin has been defined as the most active component in $\mathrm{C}$ longa and has a considerable gastroprotective and antiulcerogenic effect, its antiulcer potential activity was recently confirmed and reviewed by our laboratory [11]. Various metabolites of curcumin have been reported, including dihydrocurcumin (DHC), tetrahydrocurcumin (THC), hexahydrocurcumin (HHC), octahydrocurcumin (OHC), curcumin glucuronide, and curcumin sulphate [12]. Curcumin has a potent effect in the inhibition of matrix metalloproteinase (MMP-3) and MMP-13 gene expression by inhibiting the c-Jun-N terminal kinase (JNK), activation protein-1(AP-1), nuclear factor kappa B (NF-kB) pathways in human chondrocytes [13]. Curcumin is also known to activate and regulated endritic cells, inhibit IL-1, IL-6, and TNF- $\alpha$ along with inhibition of NF-kB activation [14]. Curcumin could prevent production of interleukin-8 (IL-8), monocyte inflammatory protein-1 (MIP-1 $\alpha$ ), monocyte chemotactic protein- 1 (MCP-1), IL-1 $\beta$, tumornecrosis factor- $\alpha$ (TNF- $\alpha$ ), 4- $\beta$ phorbor-12- $\beta$-myristate-13- $\alpha$ acetate (PMA) or lipopolysaccharide (LPS) stimulated monocytesand macrophages [15]. The antioxidant or scavenging reactive free radicals ability of curcumin arise whether from the phenolic $\mathrm{OH}$ group or from the $\mathrm{CH}_{2}$ group of the $\beta$-diketone moiety. Since, reactive free radicals can undergo electron transfer or abstract $\mathrm{H}$-atom from either of these two sites. some studies attributed the antioxidant activity to the phenolic OH group [16], however, other studies indicated that hydrogen abstraction from the methylene $\mathrm{CH}_{2}$ group is responsible for the remarkable antioxidant activity of curcumin [17]. Later, Barclay et al., by following the inhibition of styrene oxidation by a number of curcumin derivatives, suggested that curcumin is a classical phenolic chain-breaking antioxidant, donating $\mathrm{H}$ atoms from the phenolic groups not the $\mathrm{CH}_{2}$ [18]. A very important study as carried out with curcumin and dimethoxy curcumin (1,7-bis[3,4-dimethoxy phenyl]-1,6-heptadiene3,5-dione) to investigate the major function group in curcumin, the phenolic $\mathrm{OH}$ plays a major role in the activity of curcumin [19].

\section{Materials and Methods}

\subsection{Selection of the Used Doses of Curcumin}

\subsubsection{By Ulcer Lesions Count and Total Acidity}

Selection of the used doses in this study was carried out by a pioneer experiment using Shay rat model to induce the stomach ulcer in 30 rats. Afterwards the rats were randomly classified to 6 groups (5 rats for each) each group has received a selected dose of Curcumin $(0,5,10$, $20,40,80$ and $100 \mathrm{mg} / \mathrm{kg}$ ). Then the rats were scarified and samples were collected for the pioneer evaluation. The number of the ulcerative lesions in the stomach wall was counted, as well as the acid in the gastric juice was determined (Plate 1A). Among all doses 20, 40 and 80 $\mathrm{mg} / \mathrm{kg}$ of Curcumin have been selected, since, these doses seems to have anti-ulcer activity. Inclusion of the higher doses of Curcumin is due to at high concentrations, Curcumin induce bile acid secretion, which in turn one of the major ulcerogenic effectors.

\subsubsection{By Cytotoxicity and Dose Response}

Cell viability was measured as total DNA by means of the diphenylamine (DPA) assay adapted to 96-well format for robotics (Natarajan et al., 1994). The dose that produced the $50 \%$ cell survival (EC50) was extrapolated. Larger EC50 doses indicate less cytotoxicity. Freshly tissue homogenates $20 \mu \mathrm{g}$ were incubated with DPA as 

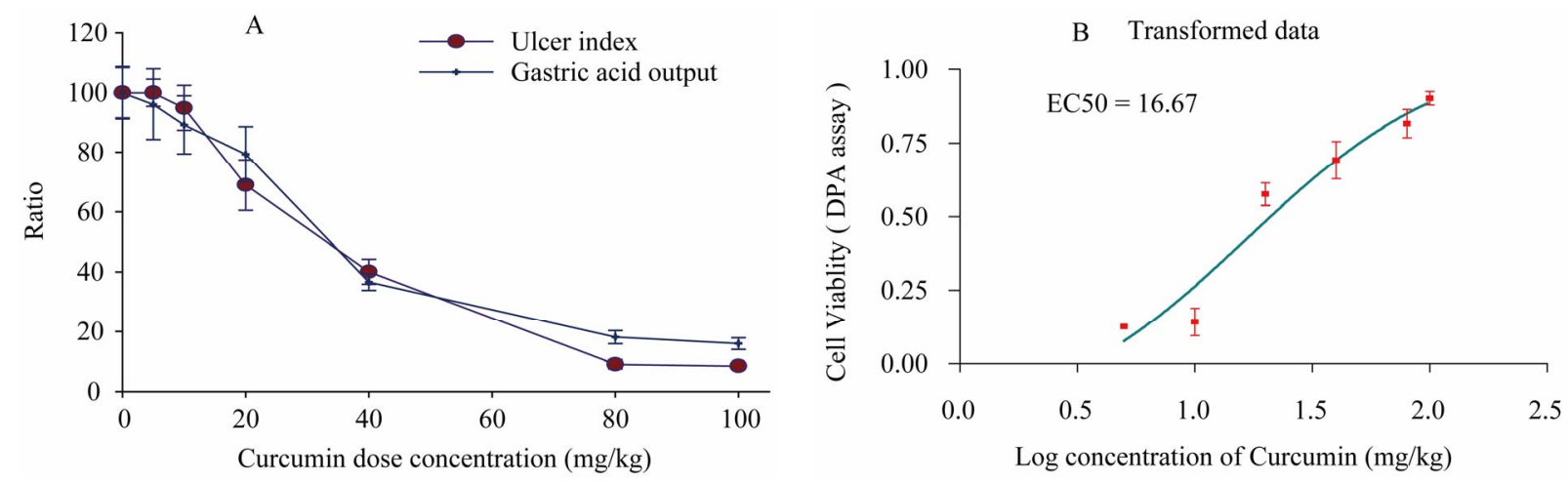

Plate 1. The percentage ratio of gastric ulcer output and ulcer index [Panel A], Cell viability measured by DNA content [Panel B] as a result of treatment with gradient doses of Curcumin measured in rats subjected to pylorus ligature (shay rat).

say, which briefly prepared by adding $80 \mu \mathrm{L}$ aliquot of a 1:5 mixture of acetaldehyde $(0.16 \%)$ and $20 \mu \mathrm{L}$ perchloric acid to $100 \mu \mathrm{L}$ of diphenylamine reagent (4\% DPA in glacial acetic acid). After $24 \mathrm{~h}$ of incubation at $37^{\circ} \mathrm{C}$, the density of the developed blue/violet color absorbance were measured at $595 \mathrm{~nm}$ minus the absorbance at 620 nm (Plate 1B).

\subsection{Animals and Treatment}

Wistar albino male rats weighing $225 \pm 25 \mathrm{~g}$ were divided into three groups $(n=10)$ and fasted for 2 days with free access to water. On the third day, the animals were deprived of water for a further $24 \mathrm{~h}$ and then underwent surgical operation after general anesthesia with Ketamine/diazepam "according to the rules of the Animal Care and Use Committee (ACUC) of the Alexandria University", Animals in group one served as a standard ulcerative control group with their pylorus ligated and had only vehicle. The animals in the second group were given curcumin at a dose of $80 \mathrm{mg} / \mathrm{kg}$ (dissolved in $0.9 \%$ $\mathrm{NaCl}$ ) after pyloric ligation. In the ulcerated omeprazole-treated therapeutic control group, omeprazole dissolved in DMSO as a single dose was administrated subcutaneously $1 \mathrm{hr}$ before surgery and after pyloric ligation at $20 \mathrm{mg} / \mathrm{kg}$. The dose of omeprazole and administration was carried out according to Warzecha et al. (2001) and Rodriguez et al. (2002), because of the impracticality of its intragastric installation. Since, PPIs are unstable at a low $\mathrm{pH}$; therefore, the oral dosage forms ("delayed release") are supplied as enteric-coated granules or tablets that dissolve only at the alkaline proximal small intestinal $\mathrm{pH}$ to prevent degradation by acid in the oesophagus and stomach.

Inclusion of a reference un-operated control group of animals and another shame-operated control group of animal was not practical since these animals will not be a real mach without pyloric ligation. The later will prevent collection of gastric juice samples and hence investigation of parameters dependent on it. The rats were killed after $19 \mathrm{~h}$ by decapitation and the gastric juice was collected and the excised stomach was then filled with 15 $\mathrm{mL}$ of $4 \%$ formalin. After $24 \mathrm{~h}$, the fixed stomach was opened along the greater curvature, gently rinsed in saline, and then pinned open to expose the gastric mucosa. The haemorrhagic and ulcerative lesions were counted and measured under a light microscope.

\subsection{Collection of Samples}

\subsubsection{Plasma}

Blood samples were collected on EDTA $(5 \mathrm{mg} / \mathrm{mL})$ by bleeding through the jugular vein into pyrogen-free polypropylene tubes, samples were mixed and centrifuged for $10 \mathrm{~min}$ at $5000 \mathrm{rpm}$ at $4{ }^{\circ} \mathrm{C}$, and were $250 \mathrm{~mL}$ aliquotted into Eppindorff's tubes and stored at $-40^{\circ} \mathrm{C}$ till used to avoid repeated freeze-thaw cycles in different assays.

\subsubsection{Juice}

After collection of blood, the animal was decapitated and the abdomen was opened. The stomach was opened along its greater curvature. The gastric content was collected in a graduated centrifuge tube. The total volume was recorded and then the juice thorough mixed and 0.5 $\mathrm{mL}$ aliquot was separated for total DNA assay and the rest of the juice was cleared of its coarse content by centrifugation at $5000 \mathrm{rpm}$ for $10 \mathrm{~min}$ at $4^{\circ} \mathrm{C}$. A total of 250 $\mathrm{mL}$ aliquots of the supernatant clear juice were allocated into Eppindorff's tubes and frozen at $-40^{\circ} \mathrm{C}$ till used.

\subsection{Measurements}

\subsubsection{Determination of Gastric Acidity}

Gastric juice samples were back titrated against $0.01 \mathrm{M}$ $\mathrm{NaOH}$ using phenolphthalein indicator $(1 \%$ in absolute ethanol) to the faint pink colored end point. The total 
acidity expressed as milliequivalents per liter $(\mathrm{mEq} / \mathrm{L})$ was calculated using the following equation.

\subsubsection{Determination of Mucosal Lesions}

The mucosal surface was washed with cold saline solution $(0.9 \mathrm{gm} \% \mathrm{NaCl})$. The mucosal ulcerations restricted to the fundic area were rapidly scored. The number of lesion bands of more than $4 \mathrm{~mm}$ length was multiplied by the severity factor 2 ; while lesions of less than $2 \mathrm{~mm}$ length had severity factor 1 . The ulcer index was calculated as the total number of lesions multiplied by the corresponding severity factor.

\subsubsection{Gastric Juice Mucin Content}

The acids in the reagent hydrolyze the complex glycoprotein of the gastric juice mucin to release $\mathrm{N}$-acetylneuraminic acid (NANA) that then reacts with the resorcinol reagent to develop the color that directly proportionates with its concentration [20]. Resorcinol Reagent prepared by adding $10 \mathrm{~mL}$ of $2 \%, \mathrm{w} / \mathrm{v}$, resorcinol in distilled water $+9.75 \mathrm{~mL}$ distilled water $+0.25 \mathrm{~mL} 0.1 \mathrm{M}$ $\mathrm{CuSO}_{4}$ in distilled water and complete to $100 \mathrm{~mL}$ with concentrated $\mathrm{HCl}$. Briefly, $20 \mu \mathrm{L}$ gastric juice or standard was added to $980 \mu \mathrm{L}$ distilled water and vortexed and keep in ice. Afterwards, $1.0 \mathrm{~mL}$ of the resorcinol reagent was added, mixed and boiled in water bath for 15 minutes. Tubes were cooled on ice for 10 minutes and 2 $\mathrm{mL}$ butyl acetate/n-butanol (v/v, 85:15) was added and vortexed for 1 minute to extract the chromogen in the upper layer. Tubes were centrifuged at $3000 \mathrm{rpm}$ for 10 minutes to clear the supernatant, and optical density (OD) of the developed color in the supernatant at $580 \mathrm{~nm}$ against water blank was recorded for samples and standards. Standards of N-acetyl-neuraminic Acid (NANA): $0.0,10.0,20.0,30.0,40.0,50.0$ and $60.0 \mathrm{ng} / \mathrm{dL}$ in distilled water. NANA concentration in samples was calculated from the standard curve.

\subsubsection{Evaluation of Gastric Mucosal Haemorrhage}

Luminal hemoglobin of the gastric juice was measured colorometrically using commercial available kit.

\subsubsection{Evaluation of the Total Peroxides Level}

Determination of the total peroxide concentration in the gastric juice and plasma were evaluated according to method described by Harma and Erel [21]. A total of 9.8 $\mathrm{mg}$ ammonium ferrous sulphate was dissolved in $10 \mathrm{~mL}$ $250 \mathrm{mM} \mathrm{H} \mathrm{SO}_{2}$. This solution was added to $90 \mathrm{~mL}$ HPLC-grade absolute methanol containing $79.2 \mathrm{mg}$ butylated hydroxytoluene. Xylenol orange $(7.6 \mathrm{mg})$ was dissolved in the solution by stirring. Blank reagent was prepared similarly with omission of the ammonium ferrous sulphate. Standard $\mathrm{H}_{2} \mathrm{O}_{2}: 10 \mu \mathrm{L}$ of $30 \%$ stock reagent was diluted in $250 \mu \mathrm{L}$ of $5 \mathrm{mM}$ PBS, pH 7.4, to get
$250 \mu \mathrm{M}$ that was $1: 1$ serially diluted in the buffer to get: $125,62.5,31.25,15.625$ and $7.8125 \mu \mathrm{M}$. Prepare fresh each time. Briefly, $50 \mu \mathrm{L}$ sample was added to $450 \mathrm{~mL}$ reagent and blank reagent in separate Eppindorff's tubes, mixed will and incubated at room temperature for $30 \mathrm{~min}$, centrifuged at $5000 \mathrm{rpm}$ for $3 \mathrm{~min}$, and $250 \mathrm{~mL}$ of the supernatant was transferred into wells of microplate and OD of the formed purple chromophore was recorded at $560 \mathrm{~nm}$ against reagent blank treated samples. Standards are treated similarly without a blank reagent treatment.

\subsubsection{Evaluation of the Total Antioxidant Capacity}

Total antioxidant capacity was measured based on the ability of the test sample to scavenge ABTS+ radical cation was compared to trolox standard [22]. $0.384 \mathrm{~g}$ of 2,2"-azino-bis[3-ethylbenz-thiazoline-6-sulfonic acid; ABTS) was dissolved in $100 \mathrm{~mL}$ deionized water to make a concentration of $7 \mathrm{mM}$. ABTS radical cation $\left(\mathrm{ABTS}^{+}\right)$ was produced by reacting ABTS stock solution with 2.45 $\mathrm{mM}$ potassium persulfate (final concentration) and allowing the mixture to stand in the dark at room temperature for $12-16 \mathrm{~h}$ before use. Afterwards, the stock solution was diluted with PBS to an absorbance of 1.6 at 415 $\mathrm{nm}$ after $1 / 50$ dilution. Then, $1.0 \mathrm{~mL}$ of diluted $\mathrm{ABTS}^{+}$ was added to $10 \mu \mathrm{L}$ of juice or plasma sample or standard or $\mathrm{H}_{2} \mathrm{O}$ as a blank, mixed, and the decrease in absorbance was recorded 6 minutes later at $415 \mathrm{~nm}$. Trolox(6-hydroxy-2,5,7,8-tetramethylchroman-2-carboxy-lic acid) was used as a standard dissolved in water at: 0.0, 0.500, 1.0, $1.5,2.0$, and $2.5 \mathrm{mM}$ concentrations. Total antioxidants concentration in samples was calculated from the standard constructed using Trolox as a standard.

\subsubsection{DNA Fragmentation (as Apoptotic Marker)}

Determination of the total and fragmented DNA in the gastric juice of the luminal content was measured colorimetrically according to method described by $\mathrm{Ku}$ rita-Ochiai et al. [23]. Hydrolysis of DNA leads to released of free deoxyribose that is colorimetrically measured after reaction with the diphenylamine reagent producing purple chromogen proportional to its concentration. Briefly, the whole juice samples were centrifuged at $5000 \mathrm{rpm}$ for $10 \mathrm{~min}$ to precipitate damaged and intact cells as a pellet from the supernatant containing fragmented DNA in gastric juice. Ice-cold trichloroacetic acid (TCA, 25\% in $\mathrm{H}_{2} \mathrm{O}_{2}$ ) was added to supernatant (1:1, $\mathrm{v} / \mathrm{v}$ ) to precipitate proteins and lipids and was centrifuged and the supernatant was recovered for assaying fragmented DNA. The pellet was resuspended in $0.5 \mathrm{~mL}$ warm TCA $\left(40^{\circ} \mathrm{C}\right)$, mixed and incubated for $10 \mathrm{~min}$ to hydrolyze intact DNA and was centrifuge to remove precipitate and supernatant was used for assay of deoxyribose released from intact DNA. Briefly, $300 \mu \mathrm{L}$ di- 
phenylamine reagent was added to $150 \mu \mathrm{L}$ supernatants/ standard deoxyribose $(0.0-3.2 \mathrm{mg} / \mathrm{L}) /$ or redistilled water blank were boiled for $10 \mathrm{~min}$ and cold on ice. The blue color developed was measured at OD $600 \mathrm{~nm}$ against reagent blank and the samples' DNA content was calculated from the standard curve. Contents in pellet and supernatant of each sample were added to get total DNA content. \% Fragmented DNA was calculated by dividing supernatant content by the total content and multiplying by 100 .

\subsubsection{Total Rate of IL-6 Content (a Pathogenic Marker)}

The IL-6 level in plasma and stomach juice was measured by ELISA according to its manufacturer's recommendations (Quantikine, cat. \# PR6000B, R \& D Systems, Inc. Minneapolis, MN, USA).

\subsubsection{Total Rate of VEGF Level (the Major Angiogenic Growth Factor)}

The level of VEGF in the plasma and in the stomach juice was evaluated by ELISA according to its manufacturer's recommendations (Quantikine Rat VEGF Immunoassay, Cat. \# RRV00, R \& D Systems, Inc., 614 McKinley Place NE, Minneapolis, MN 55413, USA).

\subsubsection{Histological Investigations}

\subsubsection{Samples Preparation for H \& E Staining}

Immediately after killing the rats under diethyl ether anesthesia, the stomach content was cleared, mucosal surface was gently washed with cold saline solution, the specimens of stomach fundic full wall of each of the four studied group were fixed in $10 \%$ formalin, embedded in paraffin wax, and paraffin sections of $5 \mu \mathrm{m}$ in thickness were prepared and stained with $\mathrm{H} \& \mathrm{E}$ for general histological examinations by Light Microscope.

\subsubsection{Samples Preparation for Toluidine Blue Staining}

5 - 8 small pieces of $1 \times 1 \mathrm{~mm}$ size were taken from the stomach fundic wall of the four studied groups, they were fixed in $5 \%$ cold buffer glutaraldehyde for one week. The specimens were then washed in phosphate buffer ( $\mathrm{pH} 7.2)-4$ changes of 15 min each with slow shaking, post fixed in $1 \%$ osmium tetroxide for $2 \mathrm{hrs}$ and washed again with phosphate buffer then were dehydrated using ascending alcoholic series at $50 \%$ for 30 $\mathrm{min}$, at $70 \%$ overnight, at $95 \%$ for $30 \mathrm{~min}$, and finally at $100 \%, 3$ changes of $30 \mathrm{~min}$ each. Samples were imbedded in propylene oxide pure for $30 \mathrm{~min}$ to remove remnants of alcohol, after those samples were imbedded in propylene oxide plus Epon $812(1: 1, \mathrm{v} / \mathrm{v})$ for 30 minutes and finally they were imbedded in pure Epon 812 for 4 hrs. Samples were finally embedded into capsules containing the embedding mixture and the tissue blocks were polymerized in an oven for 2 days at $60^{\circ} \mathrm{C}$. Semi-thin sections of $0.5 \mu \mathrm{m}$ thickness were prepared using L.K.B Ultra Microtome and were stained with toluidine blue for histopathological investigations.

\subsubsection{Statistical Analysis}

The data were expressed as Mean \pm SEM. Results were analyzed statistically using column statistics and one way ANOVA with NewmaneKeuls Multiple Comparison Test as a post test using the computer statistics Prism 3.0 package (GraphPad Software, Inc., San Diego, CA, USA). The minimum level of statistical significance was set at $P<$ 0.05 .

\section{Results}

\subsection{Effect of Different Treatments on the Ulcer Formation/Prevention and on the Total Gastric Juice Volume}

As shown in Plate 2 (Panel A), On the other hand, administration of curcumin and omeprazole significantly decreased gastric lesion formation $(P<0.001)$ in the gastric wall from $33.15 \pm 3.899$ lesions/animal in control group to $4.000 \pm 0.6602$ and to $8.215 \pm 0.8787$ lesions/ animal in treated groups with curcumin and omeprazole, respectively. Therefore, the preventive index was recorded $85.75 \pm 2.851$ and $71.71 \pm 2.697 \%$ for curcumin and omeprazole treated group, respectively. On the other hand the total gastric juice volume could be considered as a direct indicator for the secretory/anti-secretory ability of the different treatments in the stomach (plate 2, panel B). The ulcerated untreated control animals showed a total gastric juice volume of $7.280 \pm 0.7761 \mathrm{~mL} /$ animal. Most significant reduction in gastric juice volume was evident after treatment with curcumin into $3.444 \pm$ $0.5556 \mathrm{~mL} /$ animal $(P<0.01)$. Surprisingly, the Omeprazole caused a less but significant juice anti-secretory ability with juice volume of $4.850 \pm 0.6747 \mathrm{~mL} /$ animal $(P<0.05)$.

\subsection{Effect of Different Treatments on Total Gastric Juice Acidity}

As shown in Plate 2 (Panel C), the ulcerated untreated control animals showed a total gastric juice acidity of $39.60 \pm 3.384 \mathrm{mEq} / \mathrm{L}$. In comparison, gastric juice acidity was significantly reduced after treatment with curcu$\min (80 \mathrm{mg} / \mathrm{kg})$ into $6.400 \pm 0.6000 \mathrm{mEq} / \mathrm{L}(P<0.001)$. Omeprazole treatment also caused a significant reduction in total gastric acidity $10.80 \pm 1.085$. Both, curcumin and omeprazole showed potent anti-secertory abilities against gastric acid output. 

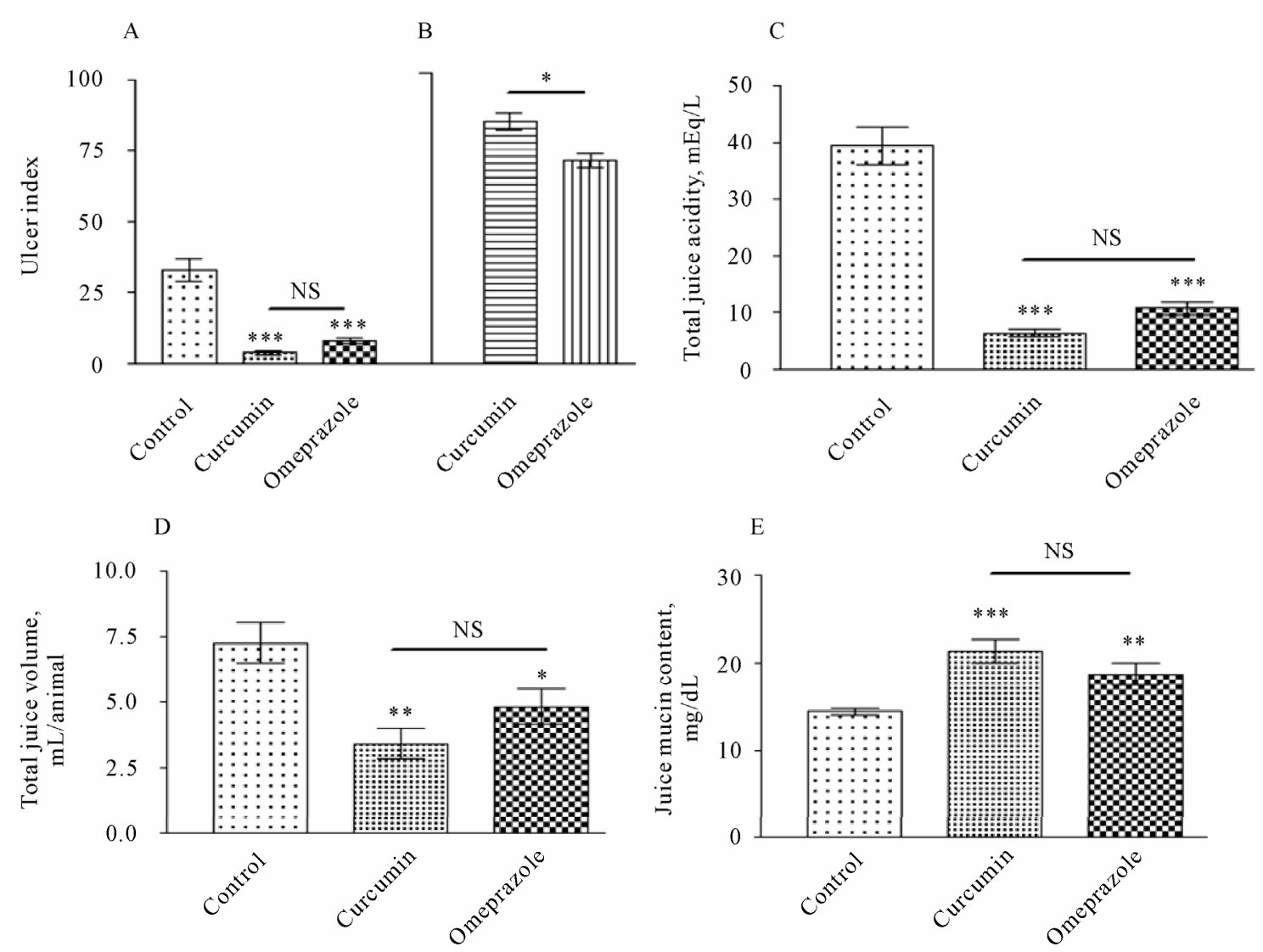

Plate 2. Ulcer index in gastric wall [Panel A] and ulcer prevention [B] gastric acid output [Panel B], gastric juice volum [panel C], and mucin content [Panel D], measured in rats subjected to pylorus ligature (shay rat).

\subsection{Effect of Different Treatments on Total Gastric Juice Mucin Content and Haemorrhage Incidence}

The increase in this biomarker is anti-ulcerogenic by being cytoprotective to gastric mucosa (Plate 2, Panel D). As shown in the ulcerated untreated control animals showed a total gastric juice mucin content of $14.410 \pm$ $0.3507 \mathrm{mg} / \mathrm{dL}$. In comparison, mucin content was most significantly increased after treatment with curcumin into $21.42 \pm 1.349 \mathrm{mg} / \mathrm{dL}(P<0.001)$, followed by omeprazole that caused a significant increase into $18.73 \pm 1.286$ $\mathrm{mg} / \mathrm{dL}(P<0.01)$. Mucosal bleeding rate was significantly increased from $1.813 \pm 0.06263$ in control group (Figure 1). As a result of the treatment the rate of mucosl bleeding was significantly decline to $0.8032 \pm 0.02516$ in curcumin treated group and to $0.7136 \pm 0.01717$ in omeprazole treated group.

\subsection{Effect of Different Treatments on the Oxidative Stress/Antioxidant}

As shown in Figure 2A, the level of the plasma total peroxide was significantly increased $(P<0.001)$ in con- trol group reached to $60.52 \pm 5.721 \mu \mathrm{M} / \mathrm{L}$, whereas, it was significantly decline to $20.33 \pm 1.642$ and $28.90 \pm$ $0.1374 \mu \mathrm{M} / \mathrm{L}$ in treated groups with curcumin and omeprazole, respectively. In gastric juice the level of total peroxide was more prominent than its level in the plasma reached to $201.8 \pm 20.76$ in the control group and significantly decreased by different treatments reached to $40.75 \pm 8.442$ and $40.85 \pm 8.452 \mu \mathrm{M} / \mathrm{L}$ in treated groups

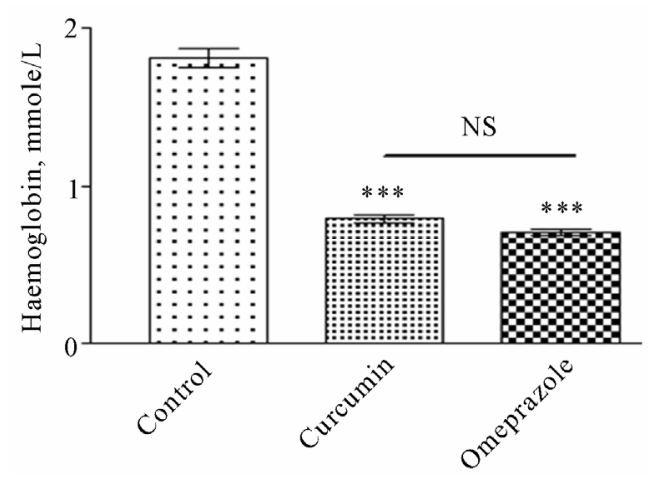

Figure 1. Mucosal bleeding rate measured in rats subjected to pylorus ligature (shay rat). 
A

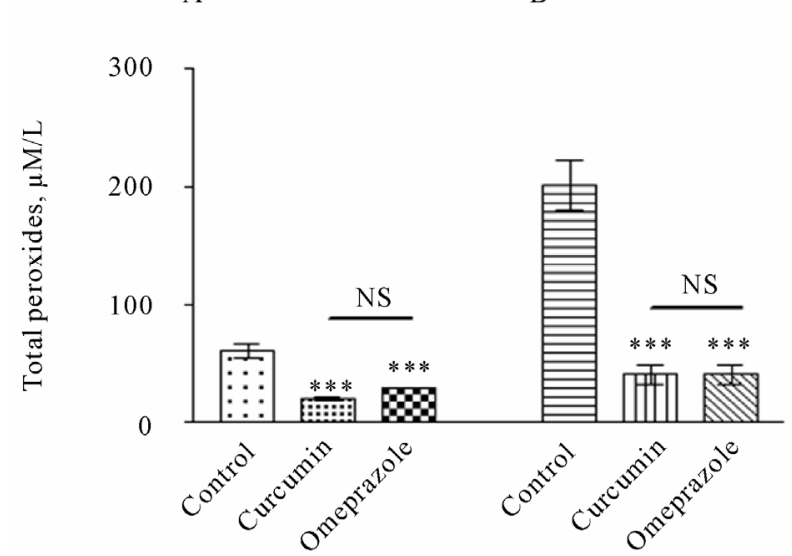

Figure 2. Total peroxides level in the gastric juice $[A]$ and plasma [B], measured in rats subjected to pylorus ligature (shay rat).

with curcumin and omeprazole, respectively (Figure 2 B).

In contrast, the total antioxidant level was significantly lower in plasma of control group $(0.7226 \pm 0.04026$ $\mathrm{mM} / \mathrm{L}$ ) than in treatment groups with curcumin or omeprazole (Figure 3A). Since, the total antioxidant capacity in the control ulcerated group was recorded $0.7959 \pm$ $0.02181 \mathrm{mM} / \mathrm{L}$. as a result of the treatment; the total antioxidant capacity was significantly increased to $1.508 \pm$ 0.09834 and to $0.9702 \pm 0.05236 \mathrm{mM} / \mathrm{L}$ in curcumin and omeprazole group, respectively. In the gastric juice (Figure 3B), the level of the total antioxidant capacity was significantly lower in the control group when compared with treated groups. Since, the level of total antioxidant in different treated groups was recorded $0.1214 \pm$ $0.009892,0.6034 \pm 0.03137$ and $0.4544 \pm 0.05306$ $\mathrm{mM} / \mathrm{L}$ for the control, curcumin and omeprazole, respectively.

\subsection{Effect of Curcumin Treatment on Apoptotic Incidence}

The level of the fragmented DNA in the supernatant of the gastric juice was recorded $0.1151 \pm 0.01458,0.02247$ \pm 0.002274 and $0.05460 \pm 0.005243$ in the control, curcumin, omeprazole, respectively (Figure 4A). In the pellet of the gastric juice, the level of the fragmented DNA was recorded $3.754 \pm 0.4589,2.090 \pm 0.2975$ and 2.443 \pm 0.2856 in the control, curcumin, omeprazole, respectively (Figure 4B). The percentage value of fragmented DNA was calculated by dividing the level of the fragmented DNA in the supernatant by its level in the pellet and multiplied with 100 (Figure 4C). The fragmented DNA level was significantly higher in control group $(27.46 \% \pm 4.101 \%)$ than in its level in treated groups re-
A

B

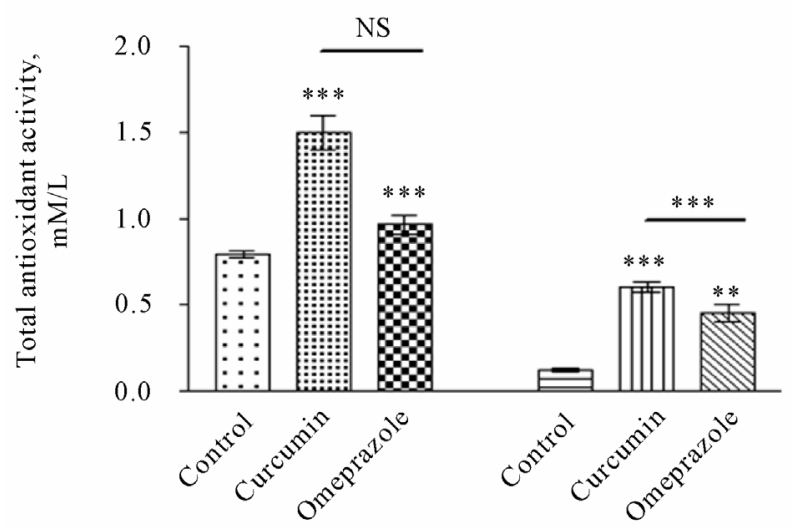

Figure 3. Total antioxidant level in the gastric juice $[\mathrm{A}]$ and plasma [B], measured in rats subjected to pylorus ligature (shay rat).

ceiving curcumin $(0.3718 \% \pm 0.06264 \%$, $)$ and omeprazole $(1.007 \% \pm 0.1458 \%)$.

\subsection{Effect of Different Treatments on the Pro-inflammatory Cytokine IL-6}

The IL-6 level was significantly higher in plasma of control group $(139.9 \pm 11.90 \mathrm{pg} / \mathrm{mL})$ than in treatment groups receiving $80 \mathrm{mg} / \mathrm{kg}$ curcumin $(59.77 \pm 5.597$ $\mathrm{pg} / \mathrm{mL})$ and $20 \mathrm{mg} / \mathrm{kg}$ omeprazole $(59.77 \pm 5.597 \mathrm{pg} / \mathrm{mL})$ (Figure 5A). If we have compared the IL-6 level in stomach with its level in the plasma we will notice that the level of IL-6 is more prominent at the stomach, where, IL-6 level was significantly higher in the gastric juice compared with its level at the plasma (Figure 5B). Since, the gastric IL-6 level was recorded $317.7 \pm 31.10 \mathrm{pg} / \mathrm{mL}$ in the control group. As a result of receiving treatments, the gastric IL-6 level was significantly reduced to $126.9 \pm$ $11.52 \mathrm{pg} / \mathrm{mL}$ and to $160.7 \pm 7.382 \mathrm{pg} / \mathrm{Ml}$, in curcumin and omeprazole group, respectively.

\subsection{Effect of Different Treatments on the Major Angiogenic Growth Factor VEGF}

As shown in Figure 6A, the VEGF level was significantly higher in plasma of control group (3.290 \pm 0.3659 $\mathrm{pg} / \mathrm{mL}$ ) than in treatment group receiving $80 \mathrm{mg} / \mathrm{kg}$ curcumin $(11.98 \pm 1.956 \mathrm{ng} / \mathrm{mL})$. While, their was no significant difference was recorded in the level of VEGF in omeprazole treated group $(5.031 \pm 0.2676 \mathrm{ng} / \mathrm{mL})$ when compared with control group. The same event was also recorded at the stomach level, since, their was no significant difference between the level of VEGF in the control group $(5.394 \pm 0.5998 \mathrm{ng} / \mathrm{Ml})$ and omeprazole treated group $(5.412 \pm 0.5073 \mathrm{ng} / \mathrm{mL})$ (Figure 6B). While, in curcumin treated group, the level of VEGF was 

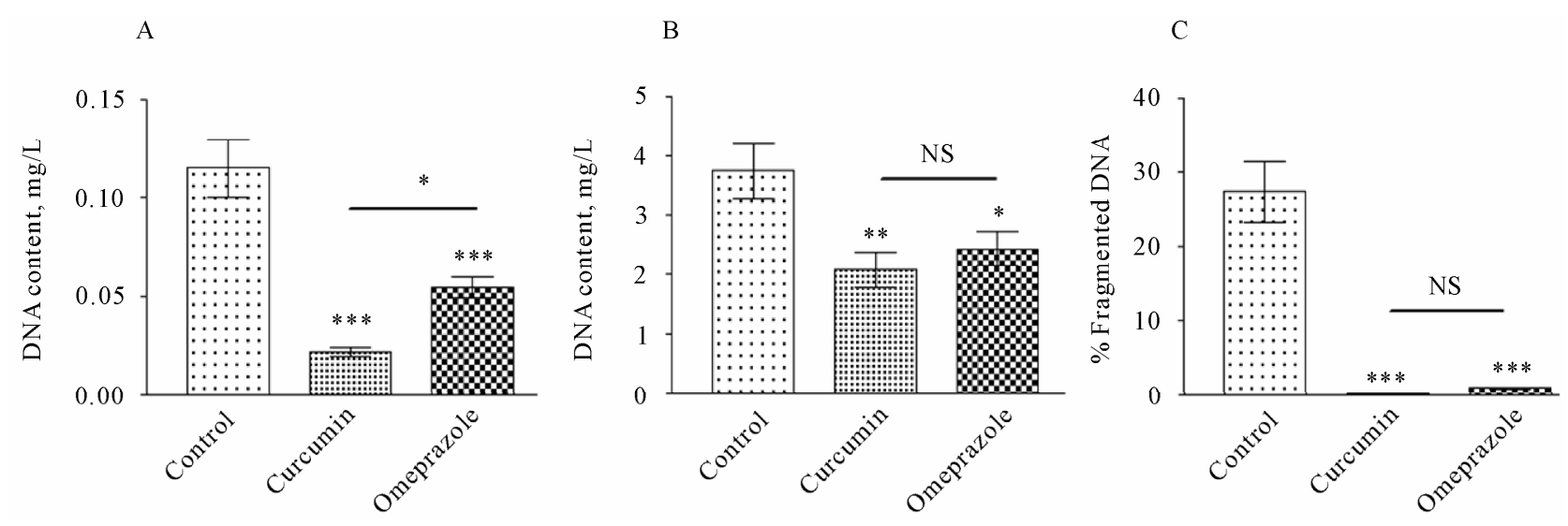

Figure 4. DNA fragmented content in the supernatant [panel A] , DNA fragmented content in the pellet [panel B] of the gastric juice and DNA fragmented percent [panel C] by dividing DNA fragmented ration by DNA fragmented ratio in the pellet multiplied by 100, measured in rats subjected to pylorus ligature (shay rat).

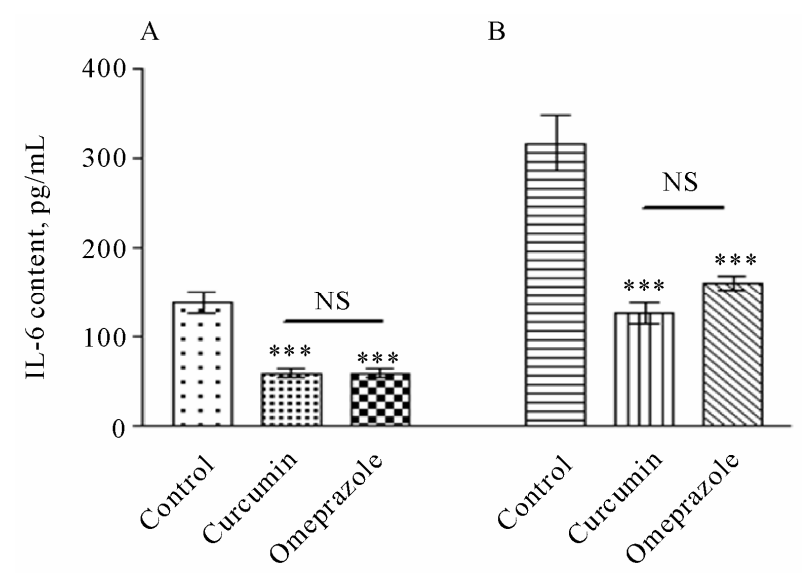

Figure 5. The level of IL-6 in the plasma [A] and in the gastric juice [B], measured in rats subjected to pylorus ligature (shay rat).

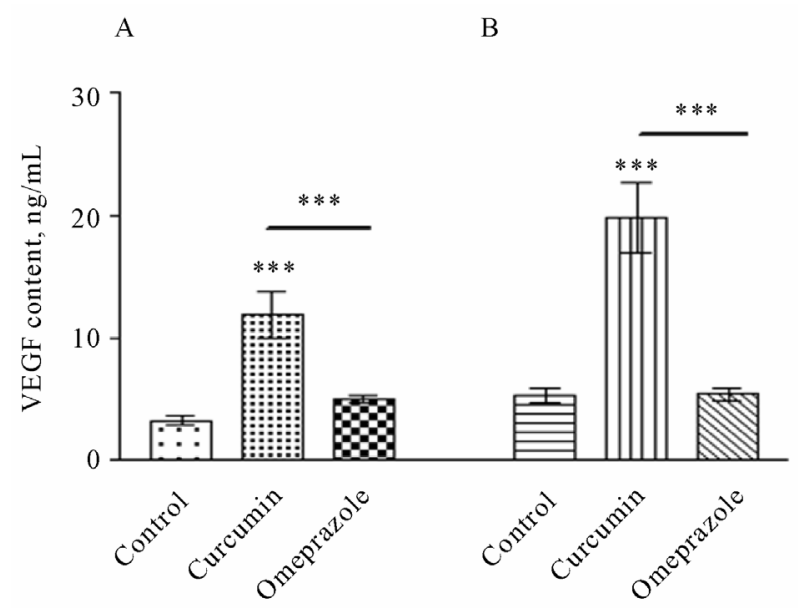

Figure 6. The level of VEGF in the plasma [A] and in the gastric juice [B], measured in rats subjected to pylorus ligature (shay rat). significantly increased to $19.88 \pm 2.900 \mathrm{ng} / \mathrm{mL}$ in comparison with each of control $(P<0.001)$ and omeprazole $(P<0.001)$ groups, respectively.

\subsection{Histopathology}

Plate 3(1): a photomicrograph in the stomach fundus of the group I positive ulcerated control "Shay Rats" showing invaded mucosa at one side by multiple lymphoid follicles with discontinuation of the lining epithelium in the affected side (arrow) while the other side looked more or less healthy $(\mathrm{H} \& \mathrm{E} \times 100)$. In the insert, a macroscopic photomicrograph of a part of the fresh stomach of positive control animals shows multiple ulcer patches (u) in the fundus, whereas, the body looked free. (2): a photomicrograph in the stomach fundus of the positive ulcerated control "Shay Rats" showing a very large sized lymph follicle extending into the submucosa, with several congested blood capillaries. Note the loss of epithelial lining at the site of invasion (arrow) $(\mathrm{H} \& \mathrm{E} \times 100)$. (3): a photomicrograph in the stomach fundus of the group I positive ulcerated control "Shay Rats" shows intact epithelial lining but markedly reduced in its thickness ((Arrow head and intact mucosa $(\mathrm{mm})(\mathrm{H} \& \mathrm{E} \times$ 200). (4) a magnified part in the stomach fundus of the group I positive ulcerated control "Shay Rats" showing apoptotic changes $(P)$ affecting the majority of cells in the lamina propria $(\mathrm{H} \& \mathrm{E} \times 1000)$.

Plate 3(5): a photomicrograph in the stomach fundus of a protective-treated ulcerated group with $\mathrm{Cn}$ showing all layers of the stomach fundus looking healthy $(\mathrm{H} \& \mathrm{E}$ $\times 100$ ). In the insert there is a macroscopic photomicrograph of a part of the fresh stomach showing the fundus and body both looking healthy. (6): a magnified part in the previous image showing a few scattered inflammatory cells (arrow) in the lamina propria (H \& E ×400). (7): a 

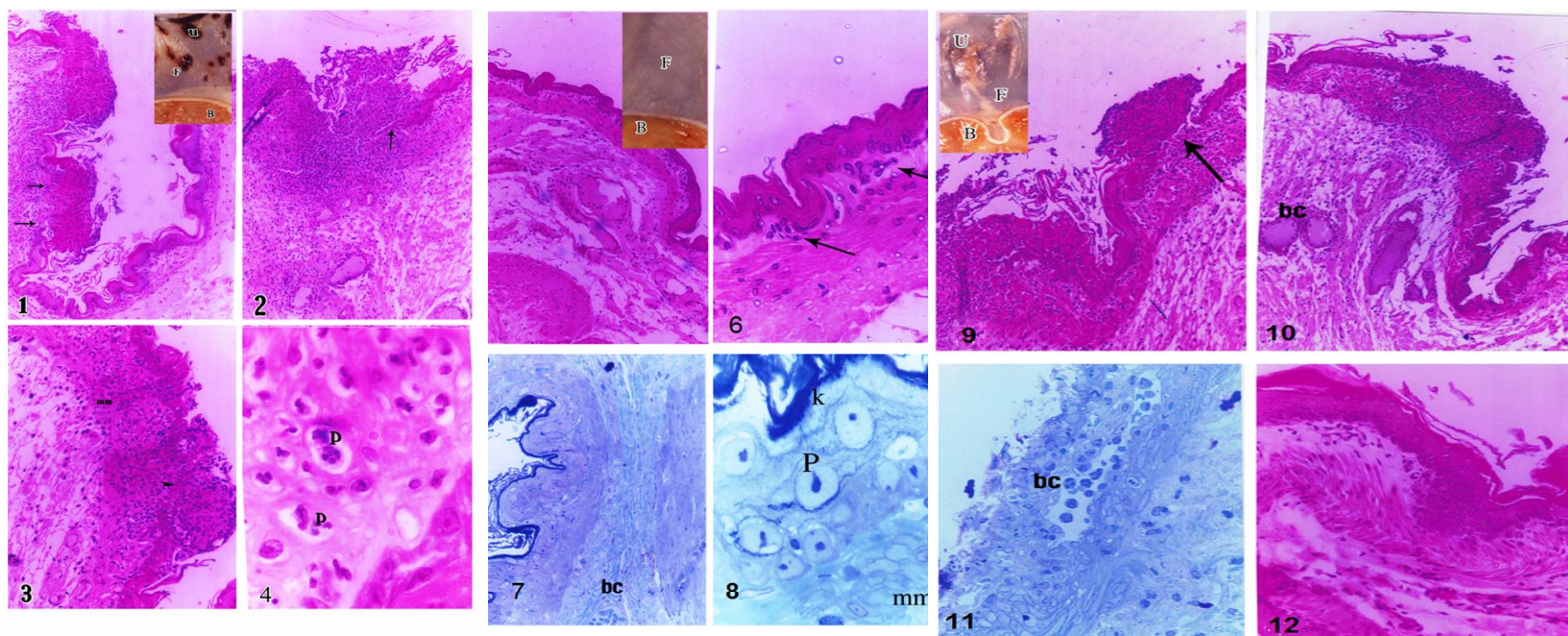

Plate 3. Histopathological changes in the fundus in rats subjected to pylorus ligature (shay rat).

photomicrograph in the stomach fundus of the group II treated ulcerated "Shay Rats" showing all layers of the stomach fundus look healthy and one blood capillary (bc) in the submucosa (toluidine blue $\times 200$ ). (8): a magnified part in the previous image showing intact keratin $(\mathrm{k})$, epithelial lining, and muscularis mucosa $(\mathrm{mm})$. The epithelial lining had minimal apoptotic changes in some cells in the form of condensed nuclear chromatin (P) (toluidine blue $\times 1000$ ).

Plate 3(9): a photomicrograph in the stomach fundus of the group IV omeprazole-treated ulcerated therapeutic control "Shay Rats" showing discontinuation of the epithelial lining associated with lymph follicle aggregation (arrow) $(\mathrm{H} \& \mathrm{E} \times 100)$. In the insert, a macroscopic photomicrograph of a part of the fresh stomach with a few ulcer patches $(\mathrm{u})$ observed in the fundus $(\mathrm{F})$, and the body (B) looked healthy. (10): a photomicrograph in the stomach fundus of the group IV omeprazole-treated ulcerated therapeutic control "Shay Rats" showing diffuse lymphocyte infiltration extending into submucosa, where dilated congested blood capillaries were present (bc) $(\mathrm{H}$ $\& \mathrm{E} \times 100)$. (11): a photomicrograph in the stomach fundus of the group IV omeprazole-treated ulcerated therapeutic control "Shay Rats" showing sloughing of almost all epithelial surface with wide irregular and congested blood capillary (bc) observed in the lamina propria (toluidine blue $\times 200$ ). (12): a photomicrograph in the stomach fundus of the group IV omeprazole-treated ulcerated therapeutic control "Shay Rats" showing all layers of fundus looking healthy $(\mathrm{H} \& \mathrm{E} \times 200)$.

\section{Discussion}

Several studies have been confirmed the antioxidant gastroprotective properties of omeprazole and strongly suggested omeprazole as a powerful antioxidant drug [24] that is beside its activity as a proton pump inhibitor. Since, omeprazole sulfenamide, besides being acid antisecretory, has "direct" and "indirect" antioxidant activity; by reacting with hypochlorous acid (HOCI) the most toxic and abundant oxidant generated by phagocytes; protonated omeprazole bind-inactivates the prooxidant effects of both free iron and copper and omeprazole inhibits neutrophil activity [25]. But, it is very important to bear that omeprazole efficiently scavenges hydroxyl radicals but not superoxide radical [26]. On the other hand, we have been confirmed the antioxidant activity of curcumin, by augmenting the level of total antioxidant and declining the level of total peroxides in treated groups [11]. Furthermore, curcumin could eliminate the hypochlorous acid (HPO) level produced by myeloperoxidase, which is the most abundant protein in neutrophils (also found in monocytes). Since, myeloperoxidase can lso use hydrogen peroxide resulting from the reduction of superoxide radicals to oxidize chloride to hypochlorous acid (HPO). A worth note is that antioxidant defence enzymes, e.g. superoxide dismutase could scavenge the super oxide radical $\mathrm{O}^{2-}$, which is responsible for lipid peroxidation, this reaction leads to an increase in the generation of peroxyl radical $\mathrm{H}_{2} \mathrm{O}_{2}$ [27], catalase and other peroxidases further reduce $\mathrm{H}_{2} \mathrm{O}_{2}^{-}$[28]. Since, the main danger for $\mathrm{H}_{2} \mathrm{O}_{2}$ arises from its ability to cross cell membranes rapidly. Once inside the cells, it can probably react with $\mathrm{Fe}^{2+}$ and $\mathrm{Cu}^{2+}$ ions to form hydroxyl radicals, which may be the origin of many toxic insults.

In this study, the gastric acidity, juice volume and the number of lesions in gastric wall of control group were significantly higher in the untreated ulcerated group; in 
contrast, the level of mucin content in the gastric juice was significantly lower in this group when compared with treated groups with either curcumin or omeprazole (Plate 2). Since, the back-diffusion of acid into the mucosa could directly lead to vascular leakage and aggressive damaging effect in the basement membrane of both epithelial and mucosal cells in the gastric wall, which could inhibit the restitution processes in the injured mucosa and induce a progression of apoptosis to deeper layers of the mucosa. Furthermore, a very brief cessation of mucosal blood flow results in a rapid decrease in the $\mathrm{pH}$ within the mucoid cap, which in turn, results in the formation of hemorrhagic erosions [29]. Thus, one could point a figure on the essential role of mucin in gastric ulcer prevention, which could prevent ulcer formation through several mechanisms, including lessening of stomach wall friction during peristalsis and gastric contractions, improving the buffering of acid gastric juice and by acting as an effective barrier to back diffusion of $\mathrm{H}^{+}$ions [30,31]. Data shown that the level of total peroxides was significantly reduced; in the contrary, total antioxidant capacity was significantly higher in the treated groups when compared with control group. Thus, the level of the fragmented DNA was significantly decline as a result of the treatment with curcumin and omeprazole. ROS such as hydrogen peroxide $\left(\mathrm{H}_{2} \mathrm{O}_{2}\right)$, hydroxyl radicals $(-\mathrm{OH})$ and superoxide anions $\left(\mathrm{O}^{2-}\right)$ have been shown to damage chromosomal DNA and other cellular components, resulting in DNA degradation and protein denaturation [32]. Data revealed increased level of the fragmented DNA which is logically resulted from the massive cellular shedding from the mucosa into the gastric lumen. And this in turn resulted in the augmentation of the fragmented DNA in untreated rats, whereas fragmented DNA was significantly reduced after treatment with curcumin and omeprazole was completely intact fundic mucosa. Their are several previous reports utilized gastric juice total DNA content as a marker for the extent of cellular death in ulceration models [33-34]. The strengthening of the mucin barrier further led to a decrease in DNA content of the gastric juice, indicating a decrease in cell shedding [35-36]. Cell shedding is an indication of integrity of the gastric mucosa. The enhanced level of cell shedding denotes the loss of integrity and decrease life span of cells, whereas decreased shedding would indicate enhanced life span of cells and promotion of defensive mechanism [34].

The massive amounts of peroxide in the control group was significantly increased that may be due to the increased level of IL-6 in the control group compared with the treated groups, particularly at the stomach level.
Since, the local inflammatory cytokine IL-6 primarily activates neutrophils, lymphocytes and monocytes/macrophages at the inflammatory site, which in turn initiates different oxidative bursts, toxic metabolites and lysosomal enzymes responsible for local tissue damage in peptic ulcer $[37,38]$. Thus, the proinflammatory IL6 could predict more precisely the severity and duration of inflammation, particularly in its acute phase, than TNF- $\alpha$ [39].

Surprisingly, the level of VEGF was significantly increased at the systemic level (in the plasma) and locally (in the stomach) only in treated group with curcumin compared with either control or omeprazole group. In experimental models of acute gastric damage, the expression of VEGF increases during healing [40], while the pre-treatment of rats with a single dose of oral VEGF exerted a protective effect against acute ethanol damage in the gastric mucosa. Furthermore, the daily administration of VEGF has been found to promote the healing of cysteamine duodenal ulcer in rats by stimulation of angiogenesis and formation of granulation tissue $[41,42]$. Since, expression of VEGF and its receptors has been demonstrated in the ulcer margin of human peptic ulcer disease [43]. In support of this notion, Wozniak et al. [2009] have been determined the role of vascular endothelial growth factor (VEGF) administered intraperitoneally in the gastroprotective response to stress-induced acute gastric ulcers in rats. A dramatic increase in the number of blood vessels was observed when VEGF was injected $24 \mathrm{~h}$ before stress exposure. Gastric secretion, depth of ulceration and ulceration index decreased significantly after VEGF application. The results demonstrate the gastroprotective effect of VEGF on stress-induced ulceration [44]. In contrast, the impairment of ulcer healing observed in ob/ob mice was characterized by reduced expression of vascular endothelial growth factor (VEGF) and impairment of angiogenesis. Systemic administration of leptin to ob/ob mice reversed the impairment of gastric ulcer healing; this reversal was accompanied by an increase in VEGF expression and angiogenesis [45]. These data suggest that VEGF might play a dual role in mucosal protection and repair. On the one hand, it might improve mucosal resistance by an increase of vascular permeability that dilutes gastrotoxic agents and reduces the area of the hemorrhagic lesions. On the other hand, it might contribute to the development of the angiogenic response together with other growth factors. Thus, VEGF is a reparative factor for ulcerated gastric mucosa through maintaining endothelial cell viability, inducing their proliferation, chemotaxis and vascular permeability [46]. 


\section{Conclusions}

Curcumin exerts its anti-ulcer activity not only by affecting oxidative stress and total antioxidant capacity but also by inhibiting IL-6 secretion and preventing apoptosis. Furthermore, curcumin promotes gastric ulcer prevention/healing by induction of angiogenesis in the granular tissue of ulcers. Further prospective studies are required to investigate the mechanism underlying the upregulation effect of curcumin on VEGF signalling pathways.

\section{REFERENCES}

[1] J. L. Wallace and M. N. Muscara, "Selective Cyclo-Oxygenase-2 Inhibitors: Cardiovascular and Gastrointestinal Toxicity," Digestive and Liver Disease, Vol. 33, Supplement 2, 2001, pp. S21-S28. doi:10.1016/S1590-8658(01)80155-9

[2] I. A. Harsch, T. Brzozowski, K. Bazela, S. J. Konturek, V. Kukharsky, T. Pawlik, E. Pawlowski, E. G. Hahn and P. C. Konturek, "Impaired Gastric Ulcer Healing in Diabetic Rats: Role of Heat Shock Protein, Growth Factors, Prostaglandins and Proinflammatory Cytokines," European Journal of Pharmacology, Vol. 481, No. 2-3, 2003, pp. 249-260. doi:10.1016/j.ejphar.2003.09.019

[3] J. R. Gum Jr., "Mucin Genes and the Proteins They Encode: Structure, Diversity, and Regulation," American Journal of Respiratory Cell and Molecular Biology, Vol. 7, No. 6, 1992, pp. 557-564.

[4] Y. Shimizu and S. Shaw, "Cell Adhesion. Mucins in the Mainstream," Nature, Vol. 366, No. 6456, 1993, pp. 630-631. doi:10.1038/366630a0

[5] U. A. Wittel, A. Goel, G. C. Varshney and S. K. Batra, "Mucin Antibodies-New Tools in Diagnosis and Therapy of Cancer," Frontiers in Bioscience, Vol. 6, 2001, pp. D1296-D1310. doi:10.2741/Wittel

[6] P. C. Konturek, T. Brzozowski, A. Duda, S. Kwiecien, S. Lober, A. Dembinski, E. G. Hahn and S. J. Konturek, "Epidermal Growth Factor and Prostaglandin E(2) Accelerate Mucosal Recovery from Stress-Induced Gastric Lesions via Inhibition of Apoptosis," Journal of Physiology-Paris, Vol. 95, No. 1-6, 2001, pp. 361-367.

[7] N. Kalia, K. D. Bardhan, M. W. Reed, S. Jacob and N. J. Brown, "Mast Cell Stabilization Prevents Ethanol-Induced Rat Gastric Mucosal Injury: Mechanisms of Protection," Journal of Gastroenterology and Hepatology, Vol. 15, No. 2, 2000, pp. 133-141. doi:10.1046/j.1440-1746.2000.02064.X

[8] K. Kohda, K. Tanaka, Y. Aiba, M. Yasuda, T. Miwa and Y. Koga, "Role of Apoptosis Induced by Helicobacter Pylori Infection in the Development of Duodenal Ulcer," Gut, Vol. 44, No. 4, 1999, pp. 456-462. doi:10.1136/gut.44.4.456

[9] W. A. Hoogerwerf and P. J. Pasricha, "Pharmacologic Therapy in Treating Achalasia," Gastrointestinal Endo- scopy Clinics of North America, Vol. 11, No. 2, 2001, pp. 311-324.

[10] D. C. Kim, S. H. Kim, B. H. Choi, N. I. Baek, D. Kim, M. J. Kim and K. T. Kim, "Curcuma Longa Extract Protects against Gastric Ulcers by Blocking $\mathrm{H}_{2}$ Histamine Receptors," Biological \& Pharmaceutical Bulletin, Vol. 28, No. 12, 2005, pp. 2220-2224. doi:10.1248/bpb.28.2220

[11] M. Tuorkey and K. Karolin, "Anti-ulcer Activity of Curcumin on Experimental Gastric Ulcer in Rats and Its Effect on Oxidative Stress/Antioxidant, IL-6 and Enzyme Activities," Biomedical and Environmental Sciences, Vol. 22, No. 6, 2009, pp. 488-495. doi:10.1016/S0895-3988(10)60006-2

[12] P. Anand, S. G. Thomas, A. B. Kunnumakkara, C. Sundaram, K. B. Harikumar, B. Sung, S. T. Tharakan, K. Misra, I. K. Priyadarsini, K. N. Rajasekharan and B. B. Aggarwal, "Biological Activities of Curcumin and Its Analogues (Congeners) Made by Man and Mother Nature," Biochemical Pharmacology, Vol. 76, No. 11, 2008, pp. 1590-1611. doi:10.1016/j.bcp.2008.08.008

[13] A. Salahuddin, A. Jeremy, J. Charles, Malemud and M. H. Tariq, "Biological Basis for the Use of Botanicals in Osteoarthritis and Rheumatoid Arthritis," Evidence-Based Complementary and Alternative Medicine, Vol. 2, No. 3 , 2005, pp. 301-308. doi:10.1093/ecam/neh117

[14] A. Vojdani and J. Erde, "Regulatory T Cells, a Potent Immunoregulatory Target for CAM Researchers: The Ultimate Antagonist (I)," Evidence-Based Complementary and Alternative Medicine, Vol. 3, No. 1, 2006, pp. 25-30. doi:10.1093/ecam/nek022

[15] Y. Abe, Hashimoto and S. T. Horie, "Curcumin Inhibition on Inflammatory Cytokine Production by Human Peripheral Blood Monocytes and Alveolar Macrophages," Pharmaceutical Research, Vol. 39, No. 1, 1999, pp. 41-47. doi:10.1006/phrs.1998.0404

[16] S. Kapoor and K. I. Priyadarsini, "Protection of Radiation-Induced Protein Damage by Curcumin," Biophysical Chemistry, Vol. 92, No. 1-2, 2001, pp. 119-126. doi:10.1016/S0301-4622(01)00188-0

[17] S. V. Jovanovic, C. W. Boone, S. Steenken, M. Trinoga and R. B. Kaskey, "How Curcumin Works Preferentially with Water Soluble Antioxidants," Journal of the American Chemical Society, Vol. 123, No. 13, 2001, pp. 3064-3068. doi:10.1021/ja003823x

[18] L. R. Barclay, M. R. Vinqvist, K. Mukai, H. Goto, Y. Hashimoto, A. Tokunaga, H. UNo, "On the Antioxidant Mechanism of Curcumin: Classical Methods are Needed to Determine Antioxidant Mechanism and Activity," Organic Letters, Vol. 2, No. 18, 2000, pp. 2841-2843. doi:10.1021/o1000173t

[19] K. I. Priyadarsini, D. K. Maity, G. H. Naik, M. S. Kumar, M. K. Unnikrishnan, J. G. Satav and H. Mohan, "Role of Phenolic O-H and Methylene Hydrogen on the Free Radical Reactions and Antioxidant Activity of Curcumin," Free Radical Biology \& Medicine, Vol. 35, No. 5, 2003, pp. 475-484. doi:10.1016/S0891-5849(03)00325-3 
[20] M. C. Plucinsky, W. M. Riley, J. J. Prorok and J. A. Alhadeff, "Total and Lipid-Associated Serum Sialic Acid Levels in Cancer Patients with Different Primary Sites and Differing Degrees of Metastatic Involvement," Cancer, Vol. 58, No. 12, 1986, pp. 2680-2685. doi:10.1002/1097-0142(19861215)58:12<2680::AID-CN CR2820581222>3.0.CO;2-L

[21] M. Harma, M. Harma and O. Erel, "Measurement of the Total Antioxidant Response in Preeclampsia with a Novel Automated Method," European Journal of Obstetrics \& Gynecology and Reproductive Biology, Vol. 118, No. 1, 2005, pp. 47-51. doi:10.1016/j.ejogrb.2004.04.012

[22] O. Erel, "A Novel Automated Method to Measure Total Antioxidant Response against Potent Free Radical Reactions," Clinical Biochemistry, Vol. 37, No. 2, 2004, pp. 112-119. doi:10.1016/j.clinbiochem.2003.10.014

[23] T. Kurita-Ochiai, K. Fukushima and K. Ochiai, "Lipopolysaccharide Stimulates Butyric Acid-Induced Apoptosis in Human Peripheral Blood Mononuclear Cells," Infection and Immunity, Vol. 67, No. 1, 1999, pp. 22-29.

[24] J. C. Becker, N. Grosser, C. Waltke, S. Schulz, K. Erdmann, W. Domschke, H. Schroder and T. Pohle, "Beyond Gastric Acid Reduction: Proton Pump Inhibitors Induce Heme Oxygenase-1 in Gastric and Endothelial Cells," Biochemical and Biophysical Research Communications, Vol. 345, No. 3, 2006, pp. 1014-1021. doi:10.1016/j.bbrc.2006.04.170

[25] D. Lapenna and F. Cuccurullo, "Hypochlorous Acid and Its Pharmacological Antagonism: An Update Picture," General Pharmacology, Vol. 27, No. 7, 1996, pp. 11451147. doi:10.1016/S0306-3623(96)00063-8

[26] W. A. Simon, E. Sturm, H. J. Hartmann and U. Weser, "Hydroxyl Radical Scavenging Reactivity of Proton Pump Inhibitors," Biochemical Pharmacology, Vol. 71, No. 9, 2006, pp. 1337-1341. doi:10.1016/j.bcp.2006.01.009

[27] D. Das, D. Bandyopadhyay, M. Bhattacharjee, R. K. Banerjee, "Hydroxyl Radical Is the Major Causative Factor in Stress-Induced Gastric Ulceration," Free Radical Biology \& Medicine, Vol. 23, No. 1, 1997, pp. 8-18. doi:10.1016/S0891-5849(96)00547-3

[28] R. S. Devi, S. Narayan, G. Vani and C. S. Shyamala Devi, "Gastroprotective Effect of Terminalia Arjuna Bark on Diclofenac Sodium Induced Gastric Ulcer," ChemicoBiological Interactions, Vol. 167, No. 1, 2007, pp. 71-83. doi:10.1016/j.cbi.2007.01.011

[29] J. L. Wallace, "Recent Advances in Gastric Ulcer Therapeutics," Current Opinion in Pharmacology, Vol. 5, No. 6, 2005, pp. 573-577. doi:10.1016/j.coph.2005.06.004

[30] L. Laine, K. Takeuchi and A. Tarnawski, "Gastric Mucosal Defense and Cytoprotection: Bench to Bedside," Gastroenterology, Vol. 135, No. 1, 2008, pp. 41-60. doi:10.1053/j.gastro.2008.05.030

[31] K. Sairam, S. Priyambada, N. C. Aryya and R. K. Goel, "Gastroduodenal Ulcer Protective Activity of Asparagus
Racemosus: An Experimental, Biochemical and Histological Study," Journal of Ethnopharmacology, Vol. 86, No. 1, 2003, pp. 1-10. doi:10.1016/S0378-8741(02)00342-2

[32] Y. Higuchi, "Chromosomal DNA Fragmentation in Apoptosis and Necrosis Induced by Oxidative Stress," Biochemical Pharmacology, Vol. 66, No. 8, 2003, pp. 1527 1535. doi:10.1016/S0006-2952(03)00508-2

[33] M. J. Tuorkey and K. K. Bdul-Aziz, "A Pioneer Study on the Anti-ulcer Activities of Copper Nicotinate Complex [CuCl(HNA)2] in Experimental Gastric Ulcer Induced by Aspirin-Pylorus [corrected] Ligation Model (Shay Model)," Biomedicine \& Pharmacotherapy, Vol. 63, No. 3, 2009, pp. 194-201. doi:10.1016/j.biopha.2008.01.015

[34] K. Mukhopadhyaya, D. Bhattacharya, A. Chakraborty, R. K. Goel and A. K. Sanyal, "Effect of Banana Powder (Musa sapientum var. paradisiaca) on Gastric Mucosal Shedding," Journal of Ethnopharmacology, Vol. 21, No. 1, 1987, pp. 11-19. doi:10.1016/0378-8741(87)90089-4

[35] A. K. Sanyal, P. K. Mitra and R. K. Goel, "A Modified Method to Estimate Dissolved Mucosubstances in Gastric Juice," Indian Journal of Experimental Biology, Vol. 21, No. 2, 1983, pp. 78-80.

[36] Y. Erbil, U. Turkoglu, U. Barbaros, E. Balik, V. Olgac, H. Kaya and B. Cimsit, "Oxidative Damage in an Experimentally Induced Gastric and Gastroduodenal Reflux Model," Surgical Innovation, Vol. 12, No. 3, 2005, pp. 219-225. doi:10.1177/155335060501200306

[37] F. S. Lehmann and G. A. Stalder, "Hypotheses on the Role of Cytokines in Peptic Ulcer Disease," European Journal of Clinical Investigation, Vol. 28, No. 7, 1998, pp. 511-519. doi:10.1046/j.1365-2362.1998.00306.x

[38] J. E. Crabtree, T. M. Shallcross, R. V. Heatley and J. I. Wyatt, "Mucosal Tumour Necrosis Factor Alpha and Interleukin-6 in Patients with Helicobacter Pylori Associated Gastritis," Gut, Vol. 32, No. 12, 1991, pp. 1473-1477. doi:10.1136/gut.32.12.1473

[39] G. Sathyanarayan, P. K. Garg, H. Prasad and R. K. Tandon, "Elevated Level of Interleukin-6 Predicts Organ Failure and Severe Disease in Patients with Acute Pancreatitis," Journal of Gastroenterology and Hepatology, Vol. 22, No. 4, 2007, pp. 550-554. doi:10.1111/j.1440-1746.2006.04752.x

[40] M. K. Jones, H. Kawanaka, D. Baatar, I. L. Szabo, K. Tsugawa, R. Pai, G. Y. Koh, I. Kim, I. J. Sarfeh and A. S. Tarnawski, "Gene Therapy for Gastric Ulcers with Single Local Injection of Naked DNA Encoding VEGF and Angiopoietin-1," Gastroenterology, Vol. 121, No. 5, 2001, pp. 1040-1047. doi:10.1053/gast.2001.29308

[41] S. Szabo, A. Vincze, Z. Sandor, M. Jadus, Z. Gombos, A. Pedram, E. Levin, J. Hagar and G. Iaquinto, "Vascular Approach to Gastroduodenal Ulceration: New Studies with Endothelins and VEGF," Digestive Diseases and Sciences, Vol. 43, No. 9, 1998, pp. 40S-45S.

[42] S. Szabo, T. Khomenko, Z. Gombos, X. M. Deng, M. R. Jadus and M. Yoshida, "Review Article: Transcription 
Factors and Growth Factors in Ulcer Healing," Alimentary Pharmacology \& Therapeutics, Vol. 14, Supplement 1, 2000, pp. 33-43.

doi:10.1046/j.1365-2036.2000.014s1033.x

[43] M. Takahashi, T. Kawabe, K. Ogura, S. Maeda, Y. Mikami, N. Kaneko, A. TeraNo and M. Omata, "Expression of Vascular Endothelial Growth Factor at the Human Gastric Ulcer Margin and in Cultured Gastric Fibroblasts: A New Angiogenic Factor for Gastric Ulcer Healing," Biochemical and Biophysical Research Communications, Vol. 234, No. 2, 1997, pp. 493-498. doi:10.1006/bbrc.1997.5974

[44] J. Wozniak-Holecka, J. Josko, M. Tyrpien, J. Kasperczyk, K. Steplewska and T. Holecki, "Influence of Vascular Endothelial Growth Factor (VEGF) on Gastroprotection in Stress-Induced Gastric Mucosal Ulcers in Rats," Methods \& Findings in Experimental \& Clinical Pharmacology, Vol. 31, No. 8, 2009, pp. 523-531.

[45] T. Tanigawa, T. Watanabe, K. Otani, Y. Nadatani, H. Machida, H. Okazaki, H. Yamagami, K. Watanabe, K. Tominaga, Y. Fujiwara, N. Oshitani, K. Higuchi and T. Arakawa, "Leptin Promotes Gastric Ulcer Healing via Upregulation of Vascular Endothelial Growth Factor," Digestion, Vol. 81, No. 2, 2010, pp. 86-95. doi: $10.1159 / 000243719$

[46] S. Milani and A. Calabro, "Role of Growth Factors and Their Receptors in Gastric Ulcer Healing," Microscopy Research and Technique, Vol. 53, No. 5, 2001, pp. 360-371. 